\title{
Indeterminate Macroscopic Satellite Nodule
}

National Cancer Institute

\section{Source}

National Cancer Institute. Indeterminate Macroscopic Satellite Nodule. NCI Thesaurus.

Code C159566.

Unable to determine if a macroscopic satellite nodule exists. 